\title{
Six of the top achievements in Canadian health research
}

\author{
Sharon E. Straus MD MSc
}

$\infty \infty$

See related articles by Straus, by Moses and by Le May

I n October 2008, the Canadian Institutes of Health Research (CIHR) and CMAJ launched a competition to acknowledge top achievements in Canadian health research (www.cihr-irsc.gc.ca/e/37888.html). Essays by the winners of the top 2 ranked achievements are available at www.cmaj.ca. Following are synopses of the other 6 winning achievements.

\section{Starting treatment of myocardial infarction sooner}

Who: Dr. Paul Armstrong is a senior cardiologist at the University Hospital and professor of medicine at the University of Alberta; Dr. Robert Welsh is chair of the Vital Heart Response Program and associate professor at the University of Alberta, Edmonton, Alberta.

What: Many regions and countries have geographic limitations that prevent immediate access to primary percutaneous coronary intervention. Dr. Armstrong and colleagues completed a feasibility study in which patients with ST-segment elevation myocardial infarction were randomly assigned within 6 hours of symptom onset to receive fibrinolysis immediately; fibrinolysis immediately plus angiography within 24 hours and rescue percutaneous coronary intervention if needed; or primary percutaneous coronary intervention immediately and a loading dose of clopidrogel. The outcomes of death, reinfarction, refractory ischemia, congestive heart failure, cardiogenic shock and major ventricular arrhythmia did not differ significantly across the 3 arms. These results suggest that early pharmacologic therapy and rescue coronary intervention may be no different than primary percutaneous coronary intervention. The results have informed larger trials evaluating this strategy and have led to the implementation of a pre-hospital care strategy for patients with ST-segment elevation myocardial infarction in Edmonton, Alberta.

\section{How the heart modulates its own workload}

Who: Dr. Adolfo de Bold is professor of pathology and laboratory medicine at the University of Ottawa Heart Institute in Ottawa, Ontario.

What: In 1982, Dr. de Bold discovered the endocrine properties of the heart and the atrial natriuretic factor. These discoveries established that the heart modulates its own workload through the secretion of hormones that help regulate blood volume and pressure. The characterization of atrial natriuretic

factor led to the identification of a new family of hormones, one of which - brain natriuretic peptide - is now used as a diagnostic test for cardiac failure. Two new drugs based on the properties of atrial natriuretic factor and brain natriuretic peptide have been the subject of recent clinical trials involving patients with heart failure. Potential future uses of the knowledge generated by Dr. de Bold's research include treatment of acute coronary syndromes, pulmonary hypertension and bronchospasm.

\section{Helping newborns breathe easier}

Who: Dr. Fred Possmayer is professor emeritus at the University of Western Ontario's Schulich School of Medicine and Dentistry in London, Ontario.

What: In the 1970s, Dr. Possmayer developed an innovative method for extracting bovine lung surfactant. Bovine lipid extract surfactant was subsequently found to be effective in substantially decreasing neonatal mortality and improving pulmonary function in infants with acute respiratory distress syndrome. Dr. Possmayer's research has led to work on optimal surfactants, and his method of extraction provides the basis for all natural surfactant preparations worldwide. The surfactant that Dr. Possmeyer developed is in widespread use in neonatal units in Canada and New Zealand and is also distributed to other parts of the world.

\section{Effectiveness of arthroscopy for osteoarthritis}

Who: Dr. Bob Litchfield is an orthopedic surgeon and is medical director at the Fowler Kennedy Sport Medicine Clinic and University of Western Ontario in London, Ontario. What: Osteoarthritis is a degenerative condition that has a substantial impact on quality of life. Although arthroscopic surgery is widely used for osteoarthritis of the knee and is one of the most common orthopedic operations performed, evidence to support its effectiveness was lacking. A recent randomized trial by Dr. Litchfield and colleagues showed that arthroscopic surgery for osteoarthritis of the knee is no better than optimized nonsurgical care. For patients, the results of this trial indicate a need to focus on nonoperative therapies.

Sharon Straus is with the LiKaShing Knowledge Institute, St. Michael's Hospital, University of Toronto, Toronto, Ont.

Cite as CMAJ 2009. DOI:10.1503/cmaj.091624 
For researchers, this trial provides an example of how highquality surgical trials can be performed. Dr. Litchfield's team proposes to use their experience to answer questions about the optimal nonoperative treatments of osteoarthritis. For the health care system, resources used in ineffective surgeries can perhaps now be directed toward more effective therapies.

\section{The evidence behind tobacco control strategies}

Who: Geoffrey Fong is professor of psychology and founder and principal investigator of the International Tobacco Control Policy Evaluation Project at the University of Waterloo in Waterloo, Ontario.

What: Professor Fong and colleagues have established the International Tobacco Control Policy Evaluation Project. This project brings colleagues from around the world together to evaluate the impact of policy-based interventions for tobacco control. Key findings from this group include the need to move to plain packaging for tobacco products. Their results also show that smoke-free legislation promotes quitting and does not cause smokers to shift smoking behaviour to home. This work has informed policy-makers as they develop and revise initiatives aimed at promoting tobacco control.
Improving recovery times after joint replacement

Who: Dr. Nizar Mahomed is head of orthopedic surgery at the Toronto Western Hospital, University Health Network in Toronto, Ontario.

What: Providing appropriate care for patients following hip and knee replacements while working toward decreasing wait lists for these procedures is a challenge in many regions and countries. Dr. Mahomed and colleagues recently completed a trial comparing inpatient and home-based rehabilitation for patients after joint replacement. They found no difference in outcomes between the 2 groups. These results were used to inform the development of a care delivery model in southern Ontario in which more patients were discharged home for rehabilitation. This model has now been expanded to include patients undergoing surgical repair of a hip fracture in order target rehabilitation therapy more appropriately.

Competing interests: Sharon Straus is the section editor of reviews at $C M A J$ but was not involved in the editorial decision-making process for this article.

Correspondence to: Dr. Sharon E. Straus, LiKaShing Knowledge Institute, St. Michael's Hospital, University of Toronto, Toronto ON M5B 1W8; fax 416 864-5805; sharon.straus@utoronto.ca 